\title{
Cardiovascular Outcomes in Systemic Lupus Erythematosus: Big Studies for Big Questions
}
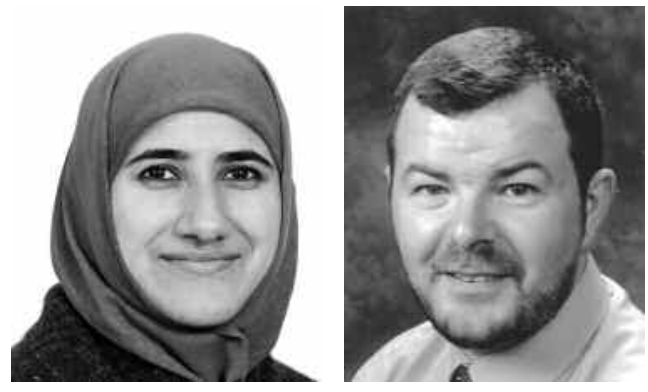

Accelerated atherosclerosis is now a well recognized complication of systemic lupus erythematosus (SLE). The overall risk of clinical coronary heart disease (CHD) in women with SLE is estimated to be between 5 and 6-fold greater than in women in the general population ${ }^{1}$. The prevalence of CHD in lupus cohorts is $6 \%-10 \%$, and this has remained constant over the last 2 decades ${ }^{1-4}$. The premature onset of CHD in SLE is also well documented, with the age of first event noted on average to be between 47 and 51 years ${ }^{1,4-6}$. Several factors contribute to clinical and subclinical CHD in SLE including "classic" Framingham risk factors and SLE-related factors. The Framingham risk factor formula has been shown not to perform as well in SLE compared to a non-SLE cohort for the prediction of coronary events ${ }^{7}$. A complex interaction involving traditional risk factors, endothelial dysfunction, systemic inflammation, and therapy such as corticosteroids, renal disease and antiphospholipid antibodies is hypothesized to result in accelerated atherosclerosis in SLE. It remains unclear to what extent these risk factors contribute to the risk of CHD and to CHD-related outcomes in SLE.

Overall survival in patients with SLE has improved substantially over the last 50 years, with 5-year survival estimates improving from 50\% in the 1950s to 93\% in 1993 in North American cohorts ${ }^{8,9}$. The improvement in survival is likely to reflect several factors. Survival rates in the general population have improved, and recognition of SLE and its complications is better and may have resulted in identifying a higher proportion of milder disease. However, it is unclear to what extent the natural history of SLE has changed over this period. Although available therapies for SLE did not change substantially in the course of the above studies, advances in our understanding of adverse effects of existing therapies such as corticosteroids are likely to have resulted in more judicious use.

In spite of these developments, there remains a significant mortality rate for patients with SLE. The 10-year survival was estimated at $85 \%$ in 1993 in a European cohort $^{10}$, and the standardized mortality ratio remains significantly increased, at about 3.0 in a recent series ${ }^{11}$. CHD remains a significant contributing factor to risk of death in SLE ${ }^{11}$. Outcomes following coronary events and factors contributing to them in SLE are therefore of much interest. However, although the relative risk of CHD is high, the absolute event rate in any single cohort will be low. For example in the most recent publication from the Toronto cohort of 1087 patients followed over an average of 8 years, 100 patients had a CHD event (about 12 per annum) ${ }^{4}$. These small absolute numbers limit the power of single-center studies to examine predictors or to describe post-CHD outcomes reliably.

In this context, Shah and colleagues report the findings of a case-control study examining in-hospital mortality and length of hospital stay following myocardial infarction (MI) from the 1993-2002 US Nationwide Inpatient Sample ${ }^{12}$. Patients with SLE were identified and compared to patients with diabetes, another condition that poses a high risk for CHD, and to patients with neither condition. The authors demonstrated an overrepresentation of males in the SLE group post-MI (22\%), relative to the 10:1 female:male baseline prevalence of SLE. This excess of CHD in men with SLE has been noted by others ${ }^{6,13}$. The adjusted hazard ratio (95\% CI) for in-hospital mortality was found to be higher in patients with SLE $(1.68,1.43-2.04)$ compared to diabetics $(1.00,0.97-1.02)$. Black men with SLE had the worst outcomes. Overall, the mean length of stay was 5.6 days, which is unsurprising if the majority of events were MI requiring reperfusion therapy; the authors used the 75th percentile as a cutoff (6 days) to define prolonged length of hospital stay. Data regarding the severity of infarctions was not reported. The odds ratio for prolonged hospitalization was higher for those with SLE $(1.48,1.32-1.79)$ compared to diabetes $(1.30,1.28-1.32)$. The investigators conclude that there is an increased risk of poor outcomes in SLE and, in this regard, SLE is comparable to diabetes. SLE patients require high priority triaging and careful post-MI followup.

See Poor outcomes after acute myocardial infarction in SLE, page 570

Personal non-commercial use only. The Journal of Rheumatology Copyright $@$ 2009. All rights reserved. 
A number of methodological issues require consideration when interpreting the findings of this study. Mortality data were recorded in hospitalized patients only and censored at the last day of hospitalization. Pre-hospital death and postdischarge data were not included, which may have resulted in an underestimation of true post-MI mortality rates. Data regarding sudden cardiac death in SLE are currently lacking, and it is unknown if its occurrence is comparable to rheumatoid arthritis (RA), where sudden cardiac death and unrecognized MI have been shown to be increased compared to non-RA subjects ${ }^{14}$. The dataset used did not allow patients to be tracked, and therefore the important question of reinfarction rates in SLE patients could not be assessed. Also SLE cases were not verified, and misclassification may have resulted in an inaccurate estimation of events. For example, it is likely that only clear cases of SLE were identified among the post-MI cohort. In contrast, there is likely to have been active screening for and thus more complete identification of diabetic cases. This would result in a bias towards overestimating SLE mortality.

The findings by Shah and colleagues are in contrast to those reported by Ward in a smaller but geographically similar population over a comparable period of time ${ }^{15}$. He demonstrated higher absolute in-hospital mortality rates for both SLE patients and controls than Shah and colleagues, but concluded there was not a significantly higher rate of adverse outcomes in SLE compared to those without SLE or those with diabetes. A number of differences between the studies may account for the differing findings. First, Ward's dataset allowed tracking of individual patients and used the 90th percentile as a cutoff for prolonged length of stay. In this situation length of hospital stay is therefore likely to reflect a more severe clinical course or complications. The mean (SD) age for both SLE patients [61 (15) yrs] and the comparison group [73 (13) yrs] was also higher in that study ${ }^{15}$. Importantly, they were able to examine coronary intervention rates and outcomes and demonstrated a decreased likelihood of coronary artery bypass graft (CABG) in SLE patients compared to those without SLE, with similar low mortality rates over a short followup period between the 2 groups following CABG. Although a few small case series have described an acceptable immediate outcome in patients with SLE undergoing coronary intervention, the long or medium-term outcome in these patients remains unknown ${ }^{15-17}$.

Neither of these studies ${ }^{12,15}$ indicated other important outcome measures, such as size or type of infarct, post-MI left ventricular function, subsequent disability, or intermediate-term mortality rates. A further key question is which contributing factors affect outcome of CHD in SLE. Therefore the influence of SLE disease-related factors, such as inflammatory disease burden or medications such as hydroxychloroquine or corticosteroids, remains unknown. Neither do we know the efficacy in SLE of usual secondary prevention measures, such as antiplatelet or lipid-lowering drugs, or angiotensin-converting enzyme inhibitors.

These studies demonstrate the challenges inherent in the study of conditions where relative rates of an outcome are high but the absolute rate of events is still low. Thirty years of studying atherosclerosis in SLE has told us that there is an excess and premature atherosclerotic burden in these patients. However, it remains unclear what the exact contributing factors are, and crucially, which interventions will be effective in primary or secondary prevention in SLE. Large-scale efforts such as the current study ${ }^{12}$ and collaborative efforts such as those undertaken by SLICC (Systemic Lupus International Collaborating Clinics) ${ }^{18}$ point the way and show that in SLE adequately powered studies are feasible. Building on these endeavors and translating current knowledge into changes in practice will be the next step. For this, the SLE community will have to think like cardiologists, in terms of the ambition and scale of studies needed to answer crucial questions.

\author{
SAHENA HAQUE, MRCP, \\ Clinical Research Fellow; \\ IAN N. BRUCE, MD, FRCP, \\ Reader and Honorary Consultant in Rheumatology, \\ ARC Epidemiology Unit, \\ University of Manchester, \\ Manchester, UK
}

Address reprint requests to Dr. Bruce.

E-mail: ian.bruce@manchester.ac.uk

\section{REFERENCES}

1. Manzi S, Meilahn EN, Rairie JE, et al. Age-specific incidence rates of myocardial infarction and angina in women with systemic lupus erythematosus: comparison with the Framingham Study. Am J Epidemiol 1997;145:408-15.

2. Petri M, Spence D, Bone LR, Hochberg MC. Coronary artery disease risk factors in the Johns Hopkins Lupus Cohort: prevalence, recognition by patients, and preventive practices. Medicine Baltimore 1992;71:291-302.

3. Gladman D, Ginzler E, Goldsmith C, et al. The development and initial validation of the Systemic Lupus International Collaborating Clinics/American College of Rheumatology damage index for systemic lupus erythematosus. Arthritis Rheum 1996;39:363-9.

4. Urowitz MB, Ibanez D, Gladman DD. Atherosclerotic vascular events in a single large lupus cohort: prevalence and risk factors. J Rheumatol 2007;34:70-5.

5. Gladman DD, Urowitz MB. Morbidity in systemic lupus erythematosus. J Rheumatol 1987;14 Suppl 13:223-6.

6. Petri M, Perez-Gutthann S, Spence D, Hochberg MC. Risk factors for coronary artery disease in patients with systemic lupus erythematosus. Am J Med 1992;93:513-9.

7. Esdaile JM, Abrahamowicz M, Grodzicky T, et al. Traditional Framingham risk factors fail to fully account for accelerated atherosclerosis in systemic lupus erythematosus. Arthritis Rheum 2001;44:2331-7.

8. Merrell M, Shulman LE. Determination of prognosis in chronic disease, illustrated by systemic lupus erythematosus. J Chronic Dis 1955;1:12-32.

9. Abu-Shakra M, Urowitz MB, Gladman DD, Gough J. Mortality

Personal non-commercial use only. The Journal of Rheumatology Copyright @ 2009 . All rights reserved. 
studies in systemic lupus erythematosus. Results from a single center. II. Predictor variables for mortality. J Rheumatol 1995;22:1265-70.

10. Tucker LB, Menon S, Schaller JG, Isenberg DA. Adult- and childhood-onset systemic lupus erythematosus: a comparison of onset, clinical features, serology, and outcome. Br J Rheumatol 1995:34:866-72.

11. Urowitz MB, Gladman DD, Tom BD, Ibanez D, Farewell VT Changing patterns in mortality and disease outcomes for patients with systemic lupus erythematosus. J Rheumatol 2008;35:2152-8.

12. Shah MA, Shah AM, Krishnan E. Poor outcomes after acute myocardial infarction in systemic lupus erythematosus. J Rheumatol 2009;36:570-5.

13. Haque S, Taylor-Fesler D, Gordon C, et al. Risk factors for clinical coronary events in patients with SLE: a multicentre case-control study [abstract]. Lupus 2007;16 Suppl:1-339.
14. Maradit-Kremers H, Crowson CS, Nicola PJ, et al. Increased unrecognized coronary heart disease and sudden deaths in rheumatoid arthritis: a population-based cohort study. Arthritis Rheum 2005;52:402-11.

15. Ward MM. Outcomes of hospitalizations for myocardial infarctions and cerebrovascular accidents in patients with systemic lupus erythematosus. Arthritis Rheum 2004;50:3170-6.

16. Bossert T, Falk V, Gummert JF, Rahmel A, Mohr FW. Coronary artery bypass grafting in patients with systemic lupus erythematosus. Z Kardiol 2003;92:219-21.

17. Bozbuga N, Erentug V, Kaya E, Akinci E, Yakut C. Coronary artery bypass grafting in patients with systemic lupus erythematosus. J Card Surg 2004;19:471-2.

18. Urowitz MB, Gladman DD. The SLICC inception cohort for atherosclerosis. Curr Rheumatol Rep 2008;104:281-5.

J Rheumatol 2009;36:467-9; doi:10.3899/jrheum.090015 